\title{
CYTOTOXIC ACTIVITY OF PLATINUM COMPLEXES CONTAINING THE ANTIOXIDANT MOIETY
}

\author{
D. Shpakovsky, T. Antonenko, R. Smirnov, Yu. Gracheva and E. Milaeva
}

Department of Chemistry, Lomonosov Moscow State University, 119991, Russia, Moscow, Leninskie gory, 1-3.

DOI: 10.19163/MedChemRussia2021-2021-533

E-mail:dmshpak@mail.ru

Platinum compounds (e.g. cisplatin, carboplatin, oxaliplatin) are widely used in cancer treatment although they severe side effects due to high toxicity and they slowly eliminated from the organism. One of the ways that helps to reduce the negative effects of anticancer drugs on healthy cells is the introduction of protective antioxidant fragments into the molecules [1-3]. It is known that 2,6-dialkylphenols are biomimetics of natural vitamins $E$ and are widely used as antioxidants.

A series of $\sigma$-aryl complexes of platinum containing fragment of 2,6-di-tert-butylphenol was synthesized. The antioxidant activity of the compounds has been studied. It was established by the CUPRAC test that R-Pt- $\mathbf{C l}$ possesses the highest ability to one-electron reduction (TEAC $=1,84 \pm 0,05$ ) compared to $\mathbf{R}-\mathrm{CH}_{3}, \mathbf{R}-\mathrm{Pt}-\mathrm{Br}$ and the standard trolox. Using the DPPH test, it was found that R-Pt- $\mathrm{SnCl}_{3}$ $\left(E C_{50}=69 \pm 4 \mu \mathrm{M}\right)$ was more active than the starting R-Pt-Cl complex $\left(\mathrm{EC}_{50}=84 \pm 5 \mu \mathrm{M}\right)$.

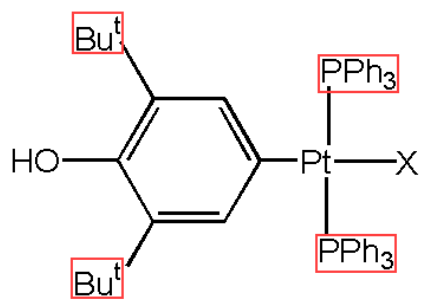

(R-Pt-X)

\section{$\mathrm{X}=\mathrm{Cl}, \mathrm{Br}, \mathrm{I}, \mathrm{SCN}, \mathrm{SnCl}_{3}$}

The cytotoxicity of the compounds R-Pt-Cl, R-Pt-Br and R-Pt-SCN was evaluated in the MTT test on human colon cancer (HCT-116), human lung cancer (A-549), human breast adenocarcinoma (MCF-7). It was found that, in contrast to cisplatin, the obtained compounds have lower $\mathrm{IC}_{50}$ values due to the trans-structure and the influence of the nature of $X$ substituent was discovered. The results open up the possibility of further investigation of trans-platinum compounds as potential anticancer drugs with a mild effect on the patient's body.

The financial support of RFBR (grant № 20-03-00471, 19-53-26002) and RSF (19-13-0084) is gratefully acknowledged.

\section{References}

[1] Shpakovsky D.B., Shtil A.A., Kharitonashvili E.V., et.al., Metallomics, 2018, 10, 406-413.

[2] T. Antonenko, D. Shpakovsky, M. Vorobyov, et.al., Applied Organometallic Chemistry, 2018, 32, e4381.

[3] E. Milaeva, Current Topics in Medicinal Chemistry, 2011, 11, 2703-2713. 